The four points therefore form a tetrahedron inscribed in the ellipsoid

$$
x^{2} / A+y^{2} / B+z^{2} / C=3 / m,
$$

and self-conjugate w.r.t. the homothetic and concentric virtual ellipsoid

$$
x^{2} / A+y^{2} / B+z^{2} / C=-1 / m ;
$$

whence we deduce that they form a circumscribed tetrahedron of the real ellipsoid

$$
\frac{x^{2}}{A}+\frac{y^{2}}{B}+\frac{z^{2}}{C}=\frac{1}{3 m}
$$

These ellipsoids are similar to Legendre's equimomental ellipsoid.

\title{
The Number of Lines that may lie upon a Surface of given Order.
}

\author{
By H. W. Richmond.
}

The greatest number of straight lines that can lie upon a surface of order $n$ (not being a ruled surface) is unknown, except if $n$ is three. Salmon and Clebsch have shown that the points of contact of lines which have a four-point contact with the surface lie upon a locus of order $n(11 n-24)$, the intersection of the surface of order $n$ with another of order $11 n-24$. Since a straight line lying wholly on the former surface must form a part of this locus, the number $n(11 n-24)$ is an upper limit to the number of lines; if $n$ is three, this gives 27, the correct number. But for values of $n>3$, it is improbable that this limit ${ }^{1}$ can be reached.

At the Colloquium, held at St Andrews in July 1930, the surfaces

$$
\text { (i) } x^{3}+y^{3}+z^{3}+t^{3}=0 \text {; (ii) } x^{4}+y^{4}=u^{4}+v^{4} \text {; }
$$

were considered, and a question was asked as to the number of lines lying on each. By a generalization of the result a theorem is obtained in regard to surfaces of any order on which lie an unexpectedly large number of lines.

"There exist surfaces of order $n$, not ruled and without conical points, on which lie $3 n^{2}$ straight lines; of these $n(n+2)$ may be real."

${ }^{1}$ See Encyklopïdie d. math. Wissenschaften, Band III, Teil 2, p. 665. 
The surface

$$
a x^{n}+b y^{n}+c z^{n}+d t^{n}=0
$$

proves the first part of the statement. The equations

$$
a x^{n}+b y^{n}=0, \quad c z^{n}+d t^{n}=0,
$$

separately represent $n$ planes, passing respectively through the lines

$$
x=y=0 \text {, and } z=t=0 .
$$

In combination they define $n^{2}$ lines of intersection of a plane of the first set with one from the second set; and all these lie on the surface. The surface therefore has upon it $n^{2}$ lines joining any one of $n$ points on $x=y=0$ to any one of $n$ points on $z=t=0$. By associating $x$ with $z$ and $y$ with $t$, or $x$ with $t$ and $y$ with $z$, we obtain two further sets of $n^{2}$ lines on the surface, $3 n^{-2}$ in all.

Few of these lines are real if $x, y, z, t$ are real planes. If $n$ is odd, three are real. If $n$ is even, eight are real when two of the constants $a, b, c, d$ are positive and two negative; otherwise none are real. A more interesting result may be derived. Let $X, Y, Z, T$ be real linear functions of the coordinates, and consider the real surface

$$
(X+i Y)^{n}+(X-i Y)^{n}=(Z+i T)^{n}+(Z-i T)^{n} .
$$

The left hand member breaks into $n$ real factors, and if equated to zero represents $n$ real planes; similarly the right hand member. The $n^{2}$ lines of intersection of these planes are real lines lying on the surface: they join $n$ real points of the line $X=Y=0$ to $n$ real points of $Z=T=0$. The other lines of the surface belong to the two systems whose equations are

(i) $(X+i Y)=a(Z+i T) ; \quad(X-i Y)=\beta(Z-i T)$ :

(ii) $(X+i Y)=a(Z-i T) ; \quad(X-i Y)=\beta(Z+i T)$ :

where $\alpha$ and $\beta$ are any $n^{\text {th }}$ roots of unity: the lines are imaginary except when $\alpha$ and $\beta$ are conjugate imaginary roots, i.e. except when $\alpha \beta=1$. The surface therefore contains $3 n^{2}$ straight lines of which $n(n+2)$ are real.

Among known quartic surfaces that of Weddle contains 25 straight lines all of which may be real; the surface has six conical points. From the foregoing work we see that the surface

$$
X^{4}-6 X^{2} Y^{2}+Y^{4}=Z^{4}-6 Z^{2} T^{2}+T^{4}
$$

contains 48 straight lines, 24 of them real and 24 imaginary. 